\title{
A wearable mixed-reality holographic computer for guiding external ventricular drain insertion at the bedside
}

\author{
Ye Li, MD, PhD, ${ }^{1}$ Xiaolei Chen, MD, PhD, ${ }^{2}$ Ning Wang, MD, PhD, ${ }^{1}$ Wenyao Zhang, PhD, ${ }^{3}$ \\ Dawei Li, MS, ${ }^{3}$ Lei Zhang, MD, ${ }^{1}$ Xin Qu, MD, ${ }^{1}$ Weitao Cheng, MD, ${ }^{1}$ Yueqiao Xu, MD, ${ }^{1}$ \\ Wenjin Chen, MD, ${ }^{1}$ and Qiumei Yang, PhD'
}

\begin{abstract}
1Department of Neurosurgery, Xuanwu Hospital, Capital Medical University; ${ }^{2}$ Department of Neurosurgery, Chinese PLA General Hospital; and ${ }^{3}$ Beijing Key Laboratory of Intelligent Information Technology, School of Computer Science, Beijing Institute of Technology, Beijing, People's Republic of China
\end{abstract}

\begin{abstract}
OBJECTIVE The goal of this study was to explore the feasibility and accuracy of using a wearable mixed-reality holographic computer to guide external ventricular drain (EVD) insertion and thus improve on the accuracy of the classic freehand insertion method for EVD insertion. The authors also sought to provide a clinically applicable workflow demonstration.
\end{abstract}

METHODS Pre- and postoperative CT scanning were performed routinely by the authors for every patient who needed EVD insertion. Hologram-guided EVD placement was prospectively applied in 15 patients between August and November 2017. During surgical planning, model reconstruction and trajectory calculation for each patient were completed using preoperative CT. By wearing a Microsoft HoloLens, the neurosurgeon was able to visualize the preoperative CT-generated holograms of the surgical plan and perform EVD placement by keeping the catheter aligned with the holographic trajectory. Fifteen patients who had undergone classic freehand EVD insertion were retrospectively included as controls. The feasibility and accuracy of the hologram-guided technique were evaluated by comparing the time required, number of passes, and target deviation for hologram-guided EVD placement with those for classic freehand EVD insertion.

RESULTS Surgical planning and hologram visualization were performed in all 15 cases in which EVD insertion involved holographic guidance. No adverse events related to the hologram-guided procedures were observed. The mean \pm SD additional time before the surgical part of the procedure began was $40.20 \pm 10.74$ minutes. The average number of passes was $1.07 \pm 0.258$ in the holographic guidance group, compared with $2.33 \pm 0.98$ in the control group $(p<0.01)$. The mean target deviation was $4.34 \pm 1.63 \mathrm{~mm}$ in the holographic guidance group and $11.26 \pm 4.83 \mathrm{~mm}$ in the control group $(p<0.01)$.

CONCLUSIONS This study demonstrates the use of a head-mounted mixed-reality holographic computer to successfully perform hologram-assisted bedside EVD insertion. A full set of clinically applicable workflow images is presented to show how medical imaging data can be used by the neurosurgeon to visualize patient-specific holograms that can intuitively guide hands-on operation. The authors also provide preliminary confirmation of the feasibility and accuracy of this hologram-guided EVD insertion technique.

https://thejns.org/doi/abs/10.3171/2018.4.JNS18124

KEYWORDS external ventricular drain; hologram; 3D Slicer; surgical planning; surgical technique

$\mathrm{B}$ ECAUSE it is considered a simple procedure, external ventricular drain (EVD) insertion is widely used to reduce intracranial hypertension for a variety of clinical indications.${ }^{6,7} \mathrm{EVD}$ insertion is often the first surgery performed by neurosurgical residents ${ }^{7}$ and generally is performed "blind" using a freehand technique based on anatomical surface landmarks. Although EVD insertion is considered a relatively simple neurosurgical technique, a nearly $50 \%$ inaccuracy rate for this procedure has been well documented in the literature. ${ }^{1,8,9}$ A possible

ABBREVIATIONS EVD = external ventricular drain.

SUBMITTED January 16, 2018. ACCEPTED April 5, 2018.

INCLUDE WHEN CITING Published online October 16, 2018; DOI: 10.3171/2018.4.JNS18124. 
explanation for this inaccuracy is that surgeons using the conventional method for EVD insertion rely to a large degree on personal experience and familiarity with neuroanatomical features, without fully taking into consideration the anatomical variations in individual patients. ${ }^{2}$ The situation becomes even more challenging in patients with aberrant anatomy due to trauma or underlying pathology. ${ }^{6}$ The need for multiple passes during EVD insertion performed with the blind freehand technique has also been criticized, ${ }^{1}$ because each pass leads to greater injury to the already compromised brain. In addition, an increased number of catheter passes is associated with a higher risk of complications, including hemorrhage and infection. ${ }^{1}$

To address the above-mentioned problems, a precise patient-tailored navigational approach would be very beneficial. ${ }^{2}$ The use of image guidance during EVD insertion has been demonstrated to greatly minimize the number of passes required for successful insertion and improve targeting accuracy. ${ }^{1,9}$ However, because currently available navigational devices are expensive and large enough to take up valuable space, $, 1,6$ image-guided methods are not routinely applied to assist in bedside EVD placement. To achieve a low-cost, portable approach, researchers have ingeniously adopted the concept of "augmented reality," in which computer-generated images are superimposed on the user's view, resulting in a composite view. With the use of smartphone software, a semitransparent 2D CT/ MR image or reconstructed model can be overlaid on the live feed from the smartphone camera during the surgical procedure. By aiming the camera at corresponding surface landmarks in the patient, the surgeon can "visualize" the internal structures of the head through the phone lens. ${ }^{2}$ A drawback of this method is that the viewing angles are confined to only a fixed direction behind the mobile phone lens, which limits enhancement of the operator's spatial perception and performance.

Mixed-reality technology is a further development that combines augmented reality and virtual reality. It uses digital objects such as holographic projections to provide virtual information in the physical environment, lending immersive realism to users' experience. The Microsoft HoloLens, a head-mounted mixed-reality holographic computer, allows operators to simultaneously maintain contact with the real and virtual worlds, and to interact with holograms according to the operators' own needs and circumstances. With the help of such state-of-the-art technology, neurosurgeons may be able to directly see highdefinition 3D holographic targets inside the patient's head and the insertion trajectory's orientation outside the head from every perspective.

In this study, we used mixed-reality technology to enhance neurosurgeons' real-time intuitive correction of their operative procedures and, theoretically, improve the spatial accuracy of EVD placement while performing EVD insertion at the bedside. To share our first hands-on experience with the use of a wearable mixed-reality holographic computer to assist bedside EVD insertion, we provide a full set of clinically applicable workflow images and a preliminary confirmation of the technical feasibility and accuracy of this method.

\section{Methods}

\section{Patient Selection}

The institutional review board of Xuanwu Hospital approved this study. As part of the consent process, we required the patient's legal decision-maker to be physically present in the hospital to obtain research consent.

Study inclusion criteria included any patient requiring ventricular drainage or intracranial pressure monitoring for any condition. Patients initially deemed to be acceptable candidates for hologram-guided EVD placement were excluded if they were judged to be too medically or neurologically at risk to allow the additional time required for $\mathrm{CT}$ acquisition and data processing.

Holographic guidance was used in 15 consecutive patients over a period of 4 months from August to November 2017. To investigate the accuracy of our new method, the same number of successive patients who underwent EVD insertion performed freehand with the classic technique made up the control group (retrospectively recruited from April to July 2017).

\section{CT Acquisition}

We routinely performed CT scanning using an 8-slice portable CT scanner (CereTom CT, NeuroLogica Corp.) before and within 48 hours after EVD placement. Imaging parameters were as follows: isotropic voxel size $5 \times$ $5 \times 5 \mathrm{~mm}$, matrix size $256 \times 256$; the imaging time was 3 minutes. In the holographic guidance group, classic localization lines were drawn before the CT scan in case of failure of holographic guidance. More importantly, several (usually more than 4) sticky electrocorticography gel electrodes were attached to the patient's cranium at sites on the forehead and parietal area along the midline, right and left Kocher's points, and above the ipsilateral tragus. These radiodense markers were used for preoperative rigid registration between the holograms and the patient's head, as well as for intraoperative registration calibration. All the DICOM data were up-sampled to $1.25 \times 1.25 \times$ $1.25-\mathrm{mm}$ voxel size at the CT console, which cost another 4 minutes. Consequently, the whole imaging duration was 7 minutes.

\section{Data Postprocessing and Holographic Visualization}

Preoperative DICOM data were imported into 3D Slicer software (version 4.7.0, nightly build, Surgical Planning Laboratory, Harvard Medical School), and segmentation of the lateral ventricles in both groups was performed to calculate the ventricular size. The surgical planning procedures in the holographic guidance group were conducted as follows. Virtual segmentation and modeling were performed for the lateral ventricles, position markers, and the patient's head. After target localization, entry point setting, and automatic trajectory calculation (including length and orientation) had been determined, a slice-wise scrutiny of the reformatted cross-sectional images perpendicular to the trajectory was performed to confirm that the planned trajectory did not include important structures where blood vessels might pass, such as the sulcus. All of the reconstructed models were imported into ParaView (version 5.4.0, www.paraview.org) for colorization 
and "transparentization." Then the data were exported and rendered in Blender (version 2.78, www.blender. org) and were saved as .fbx files recognized by the "3D viewer Beta" feature for visualization of the holograms. ${ }^{4}$ This process allowed wireless access of the data by the Microsoft HoloLens (developer's edition, Microsoft Inc.), enabling the neurosurgeon wearing the HoloLens headset to visualize holograms of the surgical plan through highdefinition lenses with small computer screens positioned in front of the eyes. An overview of the whole data postprocessing workflow is shown in Fig. 1.

\section{Hologram-Guided Operative Procedures}

All of the surgical procedures required for EVD insertion were performed by the same neurosurgeon in both patient groups. In the holographic guidance group, the neurosurgeon used the "Remote Desktop" feature in HoloLens to take control of 3D Slicer on the laptop, doublecheck the preoperative image (Fig. 2A) and surgical plan, and access detailed information such as the target point, entry point, and length of trajectory. After the holograms had been resized and rotated by gesture control or voice, the virtual markers were accurately superimposed on their physical counterparts (Fig. 2B), and the positions of the nose and ears verified the precision of registration. Thus the manual rigid coregistration between the holograms and the patient's head was accomplished (Fig. 2C and D). Then the marker on Kocher's point was removed for later scalp incision. The other 4 markers were left intact for intraoperative calibration.

After local anesthesia had been induced, a scalp incision was made. While wearing the headset (Fig. 2E), the neurosurgeon made a burr hole by using a 4-mm twist drill guided by the holographic orientation of the trajectory (Fig. 2F). If there was head movement during the drilling, the remaining markers were used for calibration, and reregistration needed to be done before the next procedure. Hologram shift was defined as intraoperative, inadvertent movement of the holograms, and a reregistration was mandatory when this occurred. The number of times that hologram shift occurred and reregistration was performed were both recorded. Aiming at the hologram of the target, the neurosurgeon intuitively guided the styletloaded catheter's insertion by keeping it aligned with the trajectory, and the process was continuously monitored during progression of the EVD placement (Fig. 2G). Both the free flow of CSF from the distal end and reaching the planned depth were considered indications of successful placement.

The neurosurgeon or his assistant noted the number of passes and the additional time required for holographic guidance. The holographic guidance time was defined as the duration of the preparation period before the surgical part of the procedure began, including imaging, surgical planning, and registration.

\section{Accuracy Evaluation}

The postoperative CT scan (Fig. 2H) from each case in both groups was imported into 3D Slicer to complete the rigid registration of the pre- and postoperative images, and target deviation was calculated. For the holographic guidance group, deviations of actual catheter tips from planned targets (point $0,0,0$ ) were visualized using the $3 \mathrm{D} \mathrm{XYZ}$ coordinate system produced in OriginPro 2016 (Origin$\mathrm{Lab}$ ). For the control group, data regarding the number of passes were collected retrospectively from the patients' medical records, which had been designed to record in detail comprehensive information for an EVD complications research study that was being conducted simultaneously in our department.

\section{Statistical Analysis}

Quantitative data are presented as means \pm SDs. The Wilcoxon rank-sum test and Fisher's exact test were applied for detection of significant differences in the demographic variables, and the paired t-test was used for comparing the ventricle size between the 2 groups. In addition, the paired t-test was used for comparing the difference in the number of passes as well as the target deviation. All statistical analyses were performed using SPSS version 20 (IBM SPSS Statistics for Macintosh, IBM Corp.), and statistical significance was set at $\mathrm{p}=0.05$.

\section{Results}

Six male and 9 female patients were included in the holographic guidance group, and their mean age was 54 \pm 14.9 years. There was no significant difference between the holographic guidance and control groups in age $(\mathrm{p}=$ $0.35)$ or sex $(p=0.27)$. In addition, no significant difference was found regarding the ventricular size between the 2 groups $(\mathrm{p}=0.36)$.

\section{Technical Feasibility}

Surgical planning and holographic visualization were achieved in all holographic guidance cases. No adverse events related to the holographic guidance procedures were observed. The mixed-reality device succeeded in creating the feeling of the actual physical presence of the computational models, whereas such an immersive holographic environment was hard to fully convey by the simultaneously recorded photos or videos. ${ }^{4}$ In addition, realtime streaming of the mixed-reality view to a computer screen allowed observers with no experience with such a technique to share the same view as the operator.

The mean additional time required for holographic guidance was $40.20 \pm 10.74$ minutes. In addition, only 1 intraoperative hologram shift was encountered during the operation for the first case in our series. Consequently, 1 more reregistration step was necessary. After drilling, $0.47 \pm 0.64$ reregistrations were needed for further calibration to ensure accuracy. According to the postoperative CT and medical records, no EVD-related complications were found in the holographic guidance group before the patients' discharge from the hospital. Table 1 summarizes the comprehensive information for each case.

\section{Technical Accuracy}

The entry point in 1 patient was modified from Kocher's point, selected during holographic planning, to 

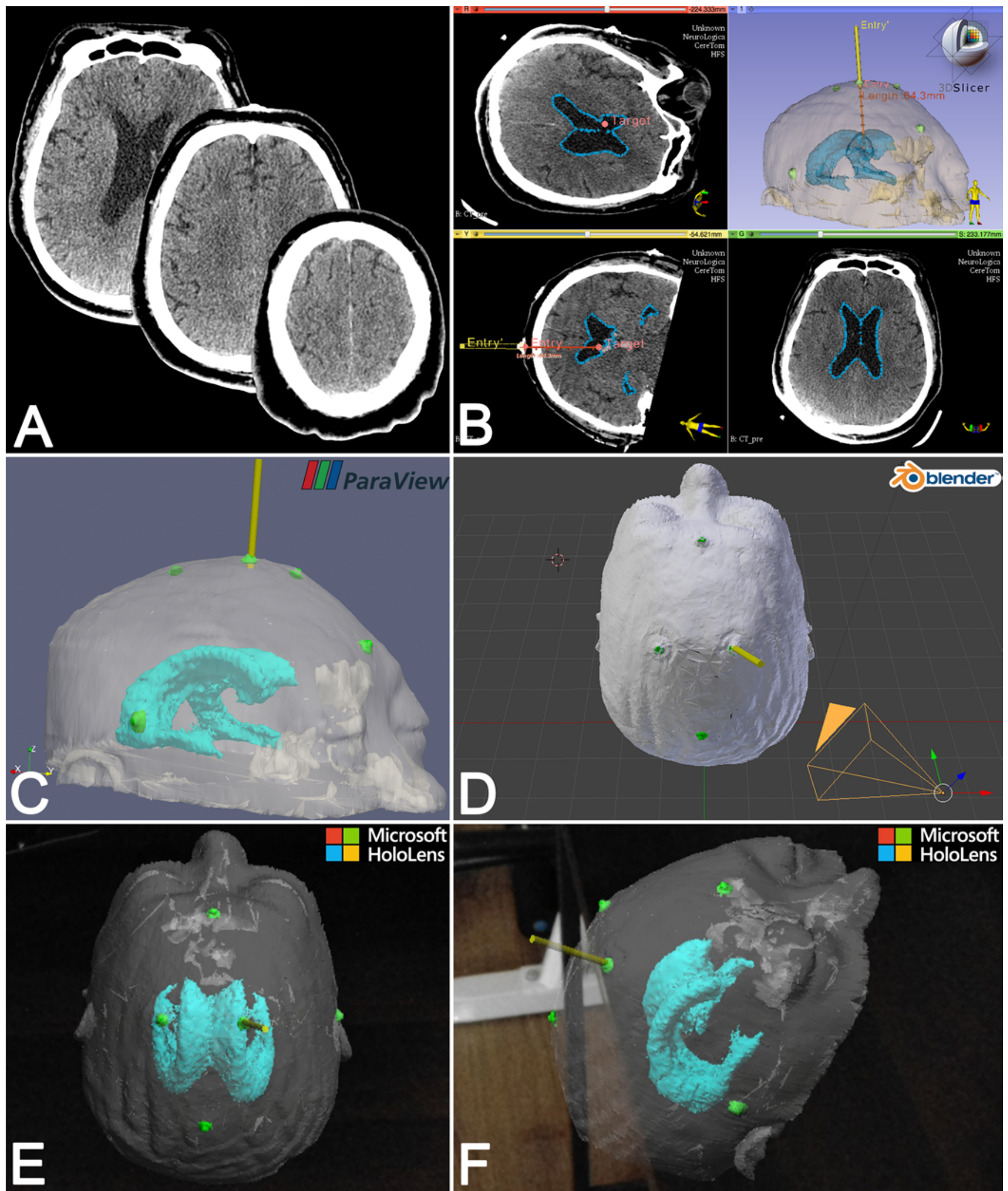

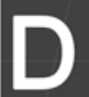

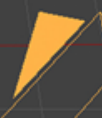

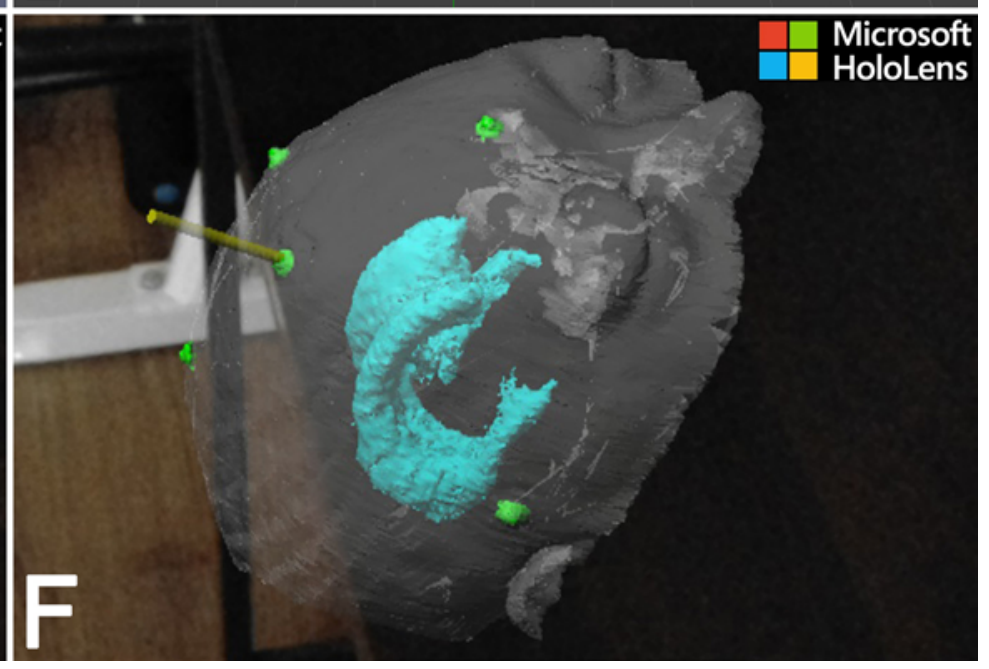

FIG. 1. A brief view of the whole data postprocessing workflow. A: Preoperative DICOM data. B: The surgical planning procedures were made in the 3D Slicer program. C: The models were imported in ParaView for colorization and transparentization. D: The data scene was rendered in Blender to initially resize and rotate the 3D models. E and F: Wearing the headset enables visualization of the holograms of the surgical plan in the physical world. Figure is available in color online only. 

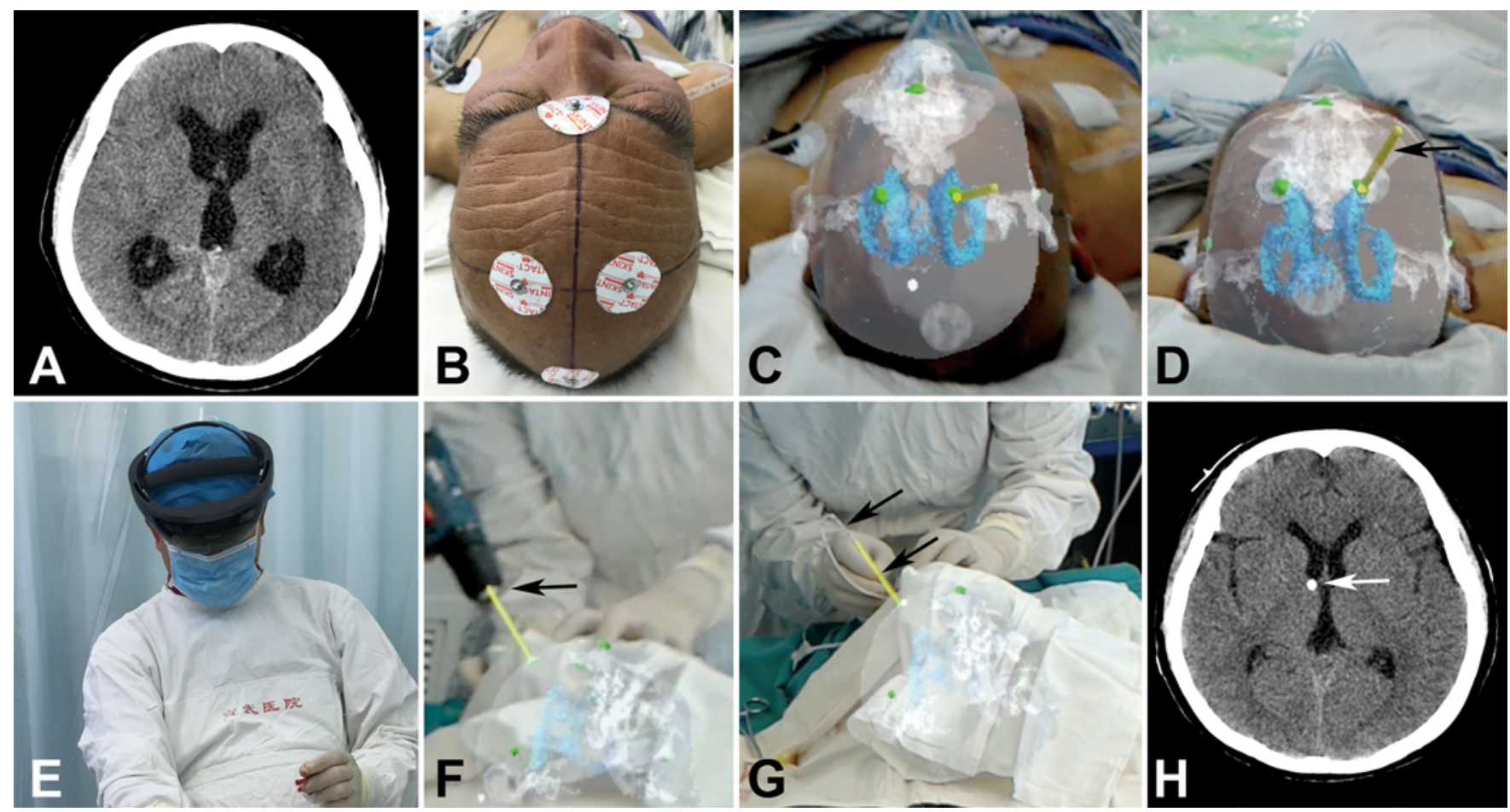

FIG. 2. Hologram-guided operation procedures. A: Preoperative CT image of a patient. B: Electrocorticography gel electrodes attached to the head of the patient as registration markers. C and D: Before (C) and after (D) manual rigid coregistration between the holograms and the patient's head. Arrow denotes hologram of the insertion trajectory. E: The neurosurgeon wore the headset during the whole procedure. F: A burr hole was performed guided by the holographic orientation of the trajectory (arrow). G: The stylet-loaded catheter (upper arrow) insertion was intuitively guided by keeping it aligned with the holographic trajectory (lower arrow). H: Postoperative CT scan verified the accuracy of EVD placement. Arrow denotes the tip of the catheter. Figure is available in color online only.

adjacent zones because a cortical vein injury through the cortical sulcus was predicted after scrutiny of each reformatted image perpendicular to the original trajectory. The number of passes was $1.07 \pm 0.258$ in the holographic guidance group, compared with $2.33 \pm 0.98$ passes in the control group $(\mathrm{p}<0.01)$.

All of the tips of inserted catheters were placed in the vicinity of the foramen of Monro in the holographic guid-

TABLE 1. Comprehensive information for 15 patients who underwent mixed-reality hologram-guided EVD insertion

\begin{tabular}{ccccccc}
\hline & \multicolumn{5}{c}{ Additional Time (mins) } & \\
Case No. & Imaging & Surgical Planning & Registration & Total & Hologram Shift & No. of Reregistration Times \\
\hline 1 & 7 & 35 & 30 & 72 & 1 & 2 \\
\hline 2 & 7 & 25 & 21 & 53 & 0 & 1 \\
\hline 3 & 7 & 21 & 11 & 39 & 0 & 0 \\
\hline 4 & 7 & 15 & 19 & 41 & 0 & 1 \\
\hline 6 & 7 & 18 & 14 & 39 & 0 & 0 \\
\hline 7 & 7 & 15 & 10 & 32 & 0 & 0 \\
\hline 8 & 7 & 13 & 13 & 33 & 0 & 1 \\
\hline 9 & 7 & 16 & 21 & 44 & 1 & 0 \\
\hline 11 & 7 & 15 & 15 & 37 & 0 & 0 \\
\hline 12 & 7 & 17 & 14 & 38 & 0 & 0 \\
\hline 13 & 7 & 15 & 9 & 31 & 0 & 1 \\
\hline 14 & 7 & 15 & 23 & 45 & 1 & 0 \\
\hline 15 & 7 & 14 & 14 & 35 & 0 & 0 \\
\hline
\end{tabular}

No EVD-related complications were found in the holographic guidance group before the patients' discharge from the hospital. 
TABLE 2. Detailed number of passes and target deviation measurement of each patient undergoing EVD insertion

\begin{tabular}{|c|c|c|c|c|c|}
\hline \multirow{2}{*}{$\begin{array}{l}\text { Case } \\
\text { No. }\end{array}$} & \multirow{2}{*}{$\begin{array}{l}\text { No. of } \\
\text { Passes }\end{array}$} & \multicolumn{4}{|c|}{ Target Deviation (mm) } \\
\hline & & Distance & $x$ & $y$ & $z$ \\
\hline 1 & 2 & 3.56 & 1.276 & 1.929 & 2.706 \\
\hline 2 & 1 & 4.158 & -2.91 & 2.159 & 2.039 \\
\hline 3 & 1 & 3.768 & 1.45 & 2.885 & 1.942 \\
\hline 4 & 1 & 3.59 & -0.384 & 0.33 & -3.554 \\
\hline 5 & 1 & 3.951 & -2.996 & 2.544 & -0.402 \\
\hline 6 & 1 & 5.643 & -2.17 & -4.599 & -2.447 \\
\hline 7 & 1 & 3.83 & -2.489 & 0.804 & -2.798 \\
\hline 8 & 1 & 4.38 & 1.768 & -0.259 & -3.999 \\
\hline 9 & 1 & 5.705 & -2.173 & 0.163 & -5.272 \\
\hline 10 & 1 & 1.139 & -0.408 & -0.617 & -0.866 \\
\hline 11 & 1 & 3.927 & -2.361 & -2.254 & -2.184 \\
\hline 12 & 1 & 4.858 & -1.357 & 2.109 & 4.161 \\
\hline 13 & 1 & 8.767 & 5.77 & -6.514 & -1.069 \\
\hline 14 & 1 & 3.236 & -1.778 & -2.53 & -0.954 \\
\hline 15 & 1 & 4.569 & 1.796 & -3.911 & 1.534 \\
\hline 16 & 2 & 8.39 & 3.99 & -7.116 & 1.959 \\
\hline 17 & 3 & 9.635 & -6.873 & 5.782 & -3.487 \\
\hline 18 & 1 & 16.961 & -15.81 & 3.776 & -4.843 \\
\hline 19 & 3 & 20.467 & 6.169 & -18.065 & 7.381 \\
\hline 20 & 2 & 15.557 & 3.933 & 9.111 & 11.981 \\
\hline 21 & 3 & 12.698 & -8.024 & 9.618 & -2.084 \\
\hline 22 & 2 & 6.029 & 3.67 & -4.569 & 1.419 \\
\hline 23 & 3 & 9.845 & -2.733 & 9.042 & -2.775 \\
\hline 24 & 3 & 7.5 & 1.209 & 4.109 & 6.157 \\
\hline 25 & 1 & 7.567 & 3.658 & -6.174 & -2.4 \\
\hline 26 & 1 & 4.848 & -3.68 & 3.036 & -0.859 \\
\hline 27 & 1 & 6.239 & -1.604 & -5.634 & -2.147 \\
\hline 28 & 4 & 18.375 & -7.904 & -8.791 & 14.068 \\
\hline 29 & 3 & 12.993 & 9.162 & -7.14 & 5.822 \\
\hline 30 & 3 & 11.743 & 2.797 & -7.345 & -8.725 \\
\hline
\end{tabular}

Cases 1-15 represent the holographically guided cases, and cases 16-30 represent the controls.

ance group. The mean target deviation was $4.34 \pm 1.63 \mathrm{~mm}$ in the holographic guidance group, whereas it was $11.26 \pm$ $4.83 \mathrm{~mm}$ in the control group $(\mathrm{p}<0.01)$. Data in Table 2 provide detailed measurements in each patient in the 2 groups, and the vectors in Fig. 3 demonstrate the actual spatial distribution of target deviation in each case, with point $0,0,0$ representing the preoperative planned target.

\section{Discussion}

The blind freehand EVD insertion technique based on superficial anatomical landmarks currently remains the method of choice due to its simplicity and, more importantly, its efficiency, ${ }^{1}$ although the rate of accurate placement is still low even when performed by neurosurgeons with adequate training and experience. ${ }^{1,8,9}$ In a multivariate logistic regression analysis, the only risk factor for inac- curate placement was the freehand technique. ${ }^{9}$ Various techniques to improve the accuracy of EVD insertion have been described in the past. However, clinicians are still reluctant to embrace these technologies for EVD placement because such devices are usually bulky, expensive, and time-consuming. ${ }^{1}$

Commercial availability of an easy-to-use 3D mixedreality device, the Microsoft HoloLens, has drawn increased attention from neurosurgeons interested in using this novel technology for bedside guidance of EVD placement. It is a self-contained holographic computer in the shape of a wireless wearable headset, ${ }^{4}$ which allows the operator freedom of head movement without losing accuracy of spatial registration. With the help of the HoloLens and previous work of other researchers, ${ }^{4}$ we have designed a system that allows us to "see through" the brain. This system not only provides a quick and portable approach to create an impression of the actual physical presence of the surgical plan, but also allows the user to interact with holograms by gesture and voice, which satisfies the aseptic demands for surgical operation. Here we provide our preliminary evidence that verifies the feasibility and accuracy of using such a bedside wearable mixed-reality holographic computer to improve the accuracy of EVD insertion.

In this study, hologram-guided EVD insertion was accomplished in every case. The neurosurgeon using this method was able to walk around the spatially fixed holograms and inspect them from every perspective. ${ }^{4}$ In addition, the safety of each holographic guidance step was verified, because no adverse events were observed. The mean additional time before the surgical part of the procedure began was 40.20 minutes; such efficiency is comparable to the use of commercially available navigation systems. ${ }^{1,6}$ With the gradual accumulation of our experience, the additional time could be shortened.

Many neurosurgeons may resist changes in the classic technique of freehand EVD placement because they see such changes as complicating a relatively simple, timeproven practice. However, our observations and those reported in other published studies implicate a substantial rate of inaccurate placement. ${ }^{8}$ In our hologram-guided series, all of the catheter tips were placed in the vicinity of the foramen of Monro, with much less mean target deviation than that which occurred in the control cases for which freehand EVD placement was used without holographic guidance. This improvement in precision demonstrated in our study is attributable to the use of individual orientation and depth of insertion determined according to the unique anatomical features of each patient, information made available to the neurosurgeon at the bedside through the use of the Microsoft HoloLens. Moreover, the difficulty in identifying surface landmarks did not cause a problem in our series because visualization of the whole head was still possible after draping. ${ }^{9}$

There always are risks involved when the EVD is passed through vital brain structures. Each additional pass may increase the frequency of axonal injury in the frontal lobes. ${ }^{1,6}$ Data also suggest that multiple passes increase the rate of hemorrhage or infection. ${ }^{6}$ In our hologram-guided series, the number of passes was minimized to 1.07 , which is comparable to results in a commercial navigation sys- 


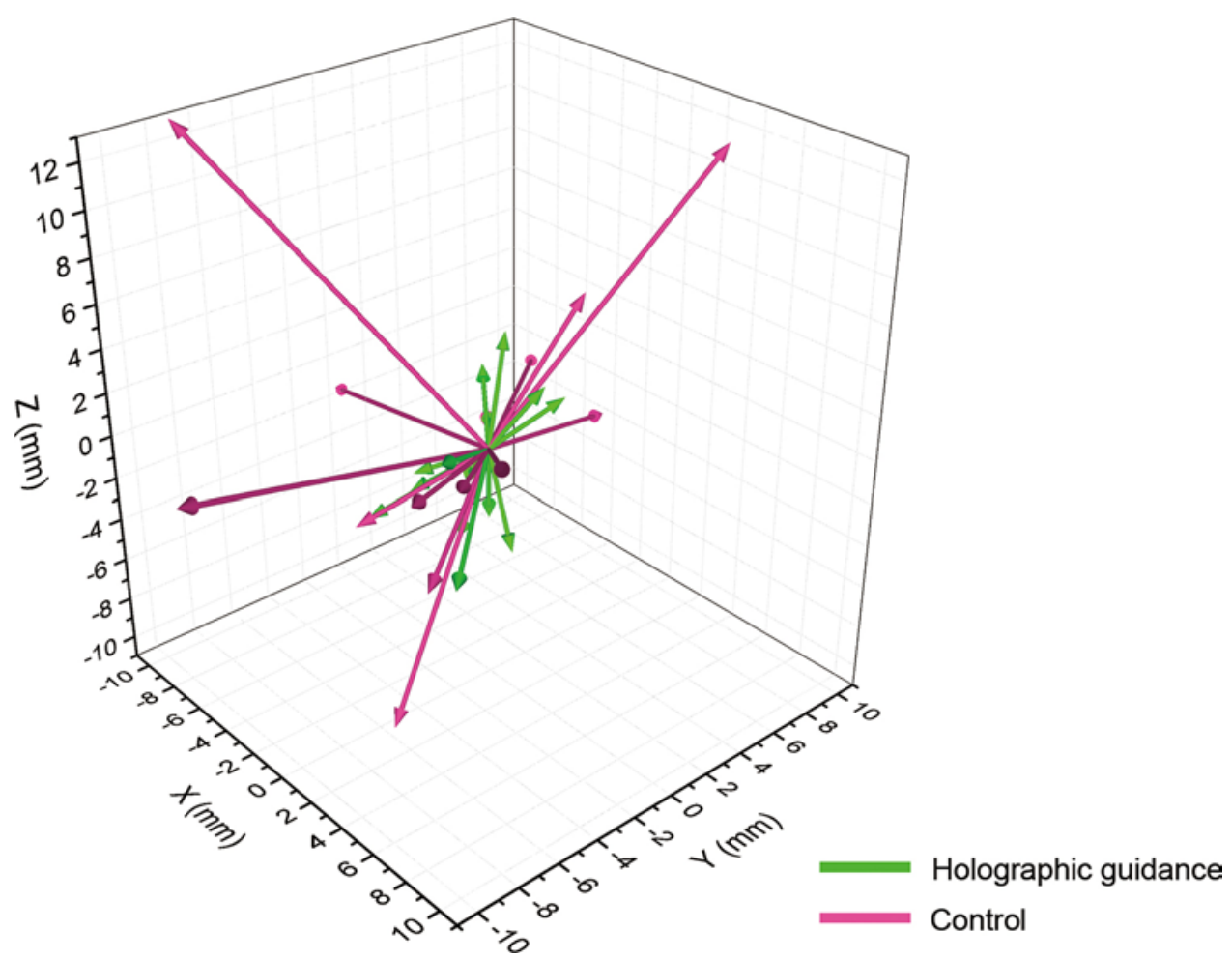

FIG. 3. Vectors representing the actual spatial distribution of the target deviation in each case. A case from the holographic guidance group (green) and a case from the control group (pink) are demonstrated. Figure is available in color online only.

tem. ${ }^{1}$ This low number of passes is a major factor contributing to the absence of complications in our study.

The successful implementation of this study is based on the 3D Slicer software. It provides a powerful, free, open-source platform for clinical research, which facilitated the development of the hologram-guided workflow in this study. ${ }^{3,5}$

\section{Limitations}

Shortcomings do exist in our research. The limited number of cases does not allow a level of evidence high enough to substantiate the accuracy of hologram-guided EVD insertion. In addition, manual registration remains the rate-determining step, and development of an automatic registration algorithm is mandatory. A better fixation method is also needed to minimize patient head movement during drilling. Moreover, we are challenged to provide enough well-trained staff to assist in the surgical planning and registration, and the use of seemingly complex technology also makes the surgeon feel mildly hesitant. These situations are always encountered in studies involving the initial clinical application of a new technique. ${ }^{6}$ However, as technology spurs accelerated improvements in medicine, neurosurgery will continue to progress by overcoming those limitations. As a matter of fact, we have already invented our own in-house software, holographic surgical planning (HSP) viewer, to advance the registration accuracy and user experience. With further improvement in registration accuracy and operation simplicity, such a technique could be used for the navigation of intracerebral hematoma aspiration and superficial tumor resection in the future.

\section{Conclusions}

To the best of our knowledge, this is the first positive experience with the use of a wearable mixed-reality holographic computer to assist neurosurgeons in bedside EVD insertion. A full set of clinically applicable workflow images is presented to demonstrate the patient-specific holographic surgical plan that intuitively guides hands-on operation. We also provide preliminary confirmation of the feasibility and accuracy of this hologram-guided EVD insertion technique.

\section{Acknowledgments}

This work was supported by grants from the National Natural Science Foundation of China (no. 81701796) and the China Scholarship Council (CSC no. 201406200059) to Ye Li.

\section{References}

1. AlAzri A, Mok K, Chankowsky J, Mullah M, Marcoux J: Placement accuracy of external ventricular drain when comparing freehand insertion to neuronavigation guidance in severe traumatic brain injury. Acta Neurochir (Wien) 159:1399-1411, 2017

2. Eftekhar B: App-assisted external ventricular drain insertion. J Neurosurg 125:754-758, 2016

3. Fedorov A, Beichel R, Kalpathy-Cramer J, Finet J, FillionRobin JC, Pujol S, et al: 3D Slicer as an image computing platform for the quantitative imaging network. Magn Reson Imaging 30:1323-1341, 2012

4. Karmonik C, Boone TB, Khavari R: Workflow for visualization of neuroimaging data with an augmented reality device. J Digit Imaging 31:26-31, 2018

5. Li Y, Zhao Y, Zhang J, Zhang Z, Dong G, Wang Q, et al: 
Low-cost interactive image-based virtual endoscopy for the diagnosis and surgical planning of suprasellar arachnoid cysts. World Neurosurg 88:76-82, 2016

6. Mahan M, Spetzler RF, Nakaji P: Electromagnetic stereotactic navigation for external ventricular drain placement in the intensive care unit. J Clin Neurosci 20:1718-1722, 2013

7. Mostofi K, Khouzani RK: Surface anatomy for implantation of external ventricular drainage: some surgical remarks. Surg Neurol Int 7 (Suppl 22):S577-S580, 2016

8. Toma AK, Camp S, Watkins LD, Grieve J, Kitchen ND: External ventricular drain insertion accuracy: is there a need for change in practice? Neurosurgery 65:1197-1201, 2009

9. Wilson TJ, Stetler WR Jr, Al-Holou WN, Sullivan SE: Comparison of the accuracy of ventricular catheter placement using freehand placement, ultrasonic guidance, and stereotactic neuronavigation. J Neurosurg 119:66-70, 2013

\section{Disclosures}

The authors report no conflict of interest concerning the materials or methods used in this study or the findings specified in this paper. 